Monográfico /Monographic

\title{
Experiencias colectivas de cuidados durante la infancia: dinámicas, debates y tensiones
}

\section{Collective caregiving practices during childhood: Dynamics, debates and tensions}

\author{
Raquel Martínez-Buján* (iD) \\ Universidade da Coruña, España \\ raquel.bujan@udc.es \\ Obdulia Taboadela Álvarez
Universidade da Coruña, España \\ obdulia.taboadela@udc.es

\section{Lucía del Moral-Espín \\ Universidad de Cádiz, España \\ lucia.delmoral@uca.es} \\ Recibido / Received: 16/01/2020 \\ Aceptado / Accepted: 10/11/2020
}

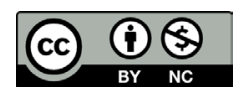

\section{RESUMEN}

El objetivo de este artículo es analizar iniciativas de cuidados de menores en A Coruña que, organizadas por los propios progenitores, trascienden de las opciones públicas y privadas del entorno. Sus resultados se basan en una investigación cualitativa realizada durante los años 2018 y 2019 en la que se han aplicado las técnicas de análisis documental, observación, entrevistas en profundidad y talleres de discusión entre las investigadoras y las participantes. En concreto, se han recogido los discursos y narraciones de 15 personas que participan en actividades de cuidados cuyo fundamento es la colaboración mutua tanto en las tareas de crianza como en su gestión. El documento explora cómo emergen estas prácticas sociales que animan a la politización colectiva de los cuidados, cuál es la filosofía que las sostiene y cuáles son sus debates internos y contradicciones.

Palabras clave: común, crianza, comunidad, reproducción social, cuidados.

\section{ABSTRACT}

This article aims to analyze the caregiving initiatives, undertaken and organized in A Coruña by the parents themselves, which transcend the public and private options available in the area. The results are based on a qualitative research project carried out in 2018 and 2019, using document analysis techniques, observation, in-depth interviews and discussion workshops between the researchers and participants. Specifically, we recorded the stories and accounts of 15 people who participated in caregiving activities based primarily on mutual collaboration in child-rearing tasks and management. This document explores the emergence of these social practices which have encouraged the collective politicization of caregiving, the philosophy behind these practices and the internal debates and contradictions that arise.

Keywords: common, community, social reproduction, caregiving.

\footnotetext{
* Autor para correspondencia / Corresponding author: Raquel Martínez-Buján, raquel.bujan@udc.es
}

Sugerencia de cita / Suggested citation: Martínez, R., Taboadela, O y del Moral-Espín, L. (2021). Experiencias colectivas de cuidados durante la infancia: dinámicas, debates y tensiones. Revista Española de Sociología, 30 (2): a31. https://doi.org/10.22325/fes/res.2021.31 


\section{INTRODUCCIÓN ${ }^{1}$}

La Crisis Financiera Global del año 2008 vino acompañada en España de una seria contestación ciudadana en forma de movimientos sociales (el movimiento de los indignados y de ocupación de las plazas, stop desahucios, Marea Verde o Yo sí Sanidad Pública son algunos ejemplos de esta réplica). Las consignas de esta respuesta cívica se centraron en críticas a la dura situación laboral y a la forma en la que se habían gestionado los bienes públicos en los años anteriores al decrecimiento económico. Se reclaman nuevas consideraciones sobre cómo debe efectuarse la gestión de estos recursos (o hetero-políticas tal y como las denominan Kioupkiolis y Pechtedelis, 2018), qué prácticas o iniciativas pueden detener la privatización de los mismos o cómo puede propiciarse la restauración de aquellos que ya se habían conseguido en años anteriores. Más allá de esta custodia ciudadana hacia los bienes públicos, entre algunos grupos sociales, se ensayan fórmulas reales de autogestión que se articulan en torno a valores colectivos y que transcienden a las habituales opciones institucionalizadas. Estas iniciativas han sido especialmente relevantes en el ámbito de la educación y la crianza infantil y surgen en medio de, al menos, dos intensos debates entrelazados entre el activismo y el terreno académico.

Por una parte, el que ha crecido en torno al marco de "lo común" (Laval y Dardot, 2015). Paradigma político que defiende la construcción de nuevas formas de gestión democrática basadas en la organización colectiva de la ciudadanía y cuyo germen contemporáneo parte de las aportaciones de Garret Hardin (1968) y Elinor Ostrom (1991) ya reformuladas por los movimientos antiglobalización y ecologistas de los años noventa. Sus reivindicaciones introducen el concepto "común" para oponerse a las tendencias de apropiación privada y ofrecer nuevas lógicas de organización social que superen los criterios individualistas y mercantiles hegemónicos (Rendueles y Subirats, 2016). En el ámbito de la educación y la crianza esto implicaría romper con las perspectivas adulto-céntricas basadas en el enfoque de la inversión social y avanzar hacia planteamientos y políticas que pongan el foco en la infancia. Es decir, hacia perspectivas que los/as reconozcan como miembros de un grupo de estatus, con capacidad de agencia y participación y que, por tanto, requieren de ciertos niveles de autonomía, reconocimiento y recursos (Daly, 2020). Por otra parte, estas iniciativas también crecen en paralelo al auge de valores feministas y de las reflexiones sobre el trabajo reproductivo y los cuidados. En este sentido, caben destacar tanto los aportes de la economía feminista y su exploración de las contradicciones existentes entre el capital y la vida (Carrasco, 2016; Pérez-Orozco, 2014), como los trabajos de Silvia Federici (2013) quien, por su parte, se ha centrado en señalar los vínculos que unen la filosofía de los "comunes" con la reproducción social. En el ámbito específico de la atención a niños/as esto se refleja en un modelo de maternidad que en los últimos tiempos parece estar recobrando fuerza bajo fórmulas y prácticas, en cierta medida ambivalentes, que pueden ser englobadas bajo el término de "maternidad intensiva" (Hays, 1996) e incluso "parentalidad intensiva" pero que, ante todo, promueven una crianza con apego que apuesta por politizar los cuidados.

En el contexto español, estos modelos se insertan en un, ya de por sí, ambivalente proceso de cambio familiar con la convivencia de expectativas contradictorias entre un ideal de familia tradicional y un evidente avance de familias con dos sustentadores en las últimas décadas. Las claves teóricas que explican esta ambivalencia "podría estar relacionada con factores de índole económica como la situación económica de las familias y el déficit de políticas familiares existentes en España" (Moreno, Ortega y Gamero, 2017, p. 162). Esta

\footnotetext{
1 Este artículo terminó de escribirse en un momento pre-expansión de la pandemia Covid-19. Las medidas adoptadas frente a ella en lo relacionado con la conciliación y la educación, refuerzan la importancia de los planteamientos teóricos y las reflexiones planteadas en el mismo.
} 
ambivalencia no contradice el contexto dibujado por los estudios sobre valores y sobre uso de tiempo que subrayan una creciente implicación paterna en el cuidado de los hijos/ as. Frente el mandato masculino tradicional, orientado hacia la provisión monetaria (y que no se abandona), ha ido aumentando la implicación y el compromiso directo y la dedicación temporal en la logística cotidiana y en lo relacional como elementos cada vez más relevantes en el rol de padre (Barbeta-Viñas y Cano, 2017). Sin embargo, el alcance real de estas trasformaciones de la paternidad ha de ser enfocado desde distintos ángulos y con perspectivas intersubjetivas. En este sentido, Flaquer et al. (2018) al analizar las últimas oleadas de la Encuesta de Empleo de Tiempo en España subrayan que, aunque ha habido un cambio importante en la composición del tiempo dedicado por los hombres al cuidado de los/as hijos/as, persiste una marcada desigualdad y especialización en determinadas tareas. Por su parte Barbeta-Viñas (2019) tras analizar de los discursos de hijos/as jóvenes sobre la implicación paterna, matiza algunas de las tesis referidas a las nuevas paternidades al percibir discordancias con los discursos de los padres, por ejemplo, en cuanto a la expansión de una paternidad más íntima, comunicativa y afectiva.

Esta conexión entre comunes, política, cuidados y modelos familiares y parentales ha sido tejida también desde una escala empírica, surgiendo como resultado de estas deliberaciones algunas iniciativas que, bajo criterios de ayuda mutua, han intentado promover espacios en los que la sostenibilidad de la vida se convierte en el eje fundamental que pauta los tiempos y los trabajos. Se trata de experiencias alternativas reales basadas en la auto-organización y que incorporan la reciprocidad en la crianza y el aprendizaje. El objetivo de este artículo es explorar cómo emergen estas prácticas culturales que animan la colaboración y participación activa de los progenitores hacia la educación durante la infancia y su politización colectiva, siendo sus contribuciones más relevantes: (i) comprender cómo estas colectividades centradas en el terreno de los cuidados y la educación generan, comparten y administran espacios, relaciones y conocimientos bajo fórmulas participativas y, (ii) explorar las interrelaciones de estas experiencias con los modelos educativos dominantes y averiguar cómo funcionan con respecto a la realidad que las rodea, lo que significa detenerse en sus debates, problemas y contradicciones.

El contexto territorial al que se circunscribe el estudio es la ciudad de A Coruña donde, durante los años 2018 y 2019, se ha realizado un trabajo de campo cualitativo dirigido a estudiar colectivos orientados a compartir la reproducción o creados para gestionar iniciativas de cuidados basadas en principios de comunalización. Las experiencias incluidas se desarrollan en un entorno urbano de tamaño pequeño y en un territorio donde el tejido asociativo ha despertado en el contexto de la crisis económica de 2008. De hecho, la base asociativa de A Coruña se ha ensanchado en los últimos años, sobre todo, a partir de la consolidación de grupos feministas con una visión crítica hacia los valores convencionales que rodean la maternidad y el surgimiento de nuevos partidos políticos que han promovido el ámbito comunitario.

Los casos que se exploran en las siguientes páginas se han construido como espacios alternativos en un principio dirigidos hacia la crianza, pero que posteriormente han dado lugar a prácticas pedagógicas que difieren de las formas tradicionales de enseñanza tanto en su versión pública como privada. Presentan, por tanto, una reflexión crítica sobre la escuela tradicional y suponen una ruptura educativa con el modelo escolar industrial pero también simbolizan la presencia y el auge de unos valores políticos que escapan del orden neoliberal capitalista y que buscan la confluencia de nuevos entornos en los que aprender valores cívicos, democráticos y participativos. No se trata de grupos con altos grados de institucionalización como los que han aparecido en otras ciudades y que ya han sido descritos en estudios previos (Keller-Garganté, 2017; Araiza y González, 2016), sino que son experiencias poco numerosas pero que reflejan un relevante cambio cultural y generacional 
sobre la maternidad-paternidad. El carácter innovador de este documento reside precisamente en que se centra en grupos de cuidados poco estructurados y territorializados en una ciudad pequeña. Ambas características, a nuestro entender, posibilitan que la evolución de dichos espacios no se rija tanto sobre los valores que representan, sino sobre cómo pueden cooperar con el entorno en el que habitan (especialmente a través de las escuelas) para convertirse en fuerzas socialmente transformadoras.

Para ello, el texto se divide en los siguientes apartados. Primero, se aborda teóricamente el marco de "lo común" y de la reproducción social desde un enfoque feminista con el fin de conceptualizar las posibilidades de la comunidad como una agencia alternativa desde la que organizar los cuidados. También se estudian las filosofías que sobre maternidades y crianza animan estas prácticas colectivas. Segundo, se detalla el diseño metodológico de la investigación. Tercero, se explora el contexto en el que emergen estas iniciativas, sus fórmulas participativas de funcionamiento y las rupturas que representan con respecto al modelo convencional de cuidados. Cuarto, se indaga en los debates y contradicciones de estas iniciativas y las relaciones que mantienen con el sistema formal de educación. Finalmente, se ofrecen unas conclusiones.

\section{COMUNES, REPRODUCCIÓN SOCIAL, MATERNIDADES Y CRIANZA}

El activismo político vivido en las calles a raíz de la crisis económica y sus devastadores efectos sobre la destrucción de empleo y la aminoración de la protección social pública inundó también a la academia, la cual, se nutrió de interesantes conexiones con estos movimientos sociales. En el ámbito de la organización social del cuidado las reflexiones más potentes se activan desde las aportaciones feministas porque en este contexto social retoman el concepto de comunidad en el sentido que había sido otorgado por las corrientes feministas-marxistas de los años 70 de la mano de autoras como María Rosa Dalla Costa y Selma James (1972). En su trabajo seminal titulado "El poder de la mujer y la subversión de la comunidad" ya explicaban cómo el capitalismo fabricó un modelo específico de familia doméstica y de mujer-ama de casa cuya finalidad consistía en desempeñar gratuitamente y de manera aislada las necesidades socialmente necesarias para la reproducción de la fuerza de trabajo. La lucha feminista era el camino para revertir este orden privado que instrumentalizaba el trabajo de las mujeres; la vía para hacerlo era politizarlo y desplazarlo hacia afuera reapropiándose de él en un sentido colectivo.

La recuperación de este debate por parte de la economía feminista ha sido esencial para conectar las posiciones teóricas con las prácticas aplicadas de los cuidados en la comunidad. Sin esta revisión del feminismo clásico no podría superarse la visión de la "comunidad" como una esfera delegada a las mujeres bajo la que se refuerza su posición como cuidadoras en el espacio doméstico. De hecho, éste había sido el enfoque predominante durante los años 80 porque las políticas públicas orientadas al bienestar, si bien, habían intentado reforzar la atención social en los domicilios, relegaron esta responsabilidad en las mujeres y en las redes sociales vecinales (amigas, vecinas, voluntarias...) (Vega y Martínez-Buján, 2017). Ello supuso duras críticas desde los estudios feministas de esa década, los cuales, demostraron como el cuidado dentro de la comunidad así entendida no apoyaba la autonomía, sino que más bien acentuaba la dependencia de las mujeres respecto del ámbito del hogar (Graham, 1997). Esta orientación de la protección social hacia lo que se ha denominado como "community care", si bien se consolidó como filosofía de algunas prestaciones ampliamente reconocidas en los países europeos, como es el caso de los servicios de atención a domicilio, experimentó un fuerte retroceso a partir del giro asistencialista de las políticas neoliberales instauradas en los años ochenta. Éstas 
limitaron el apoyo público, el cual pasaría a pensarse como una ayuda residual (nuevamente conectada con los sectores más vulnerables) (Gelb, 1988, p. 59).

En este contexto de revisión de posiciones teóricas, autoras feministas como Sandra Harding comienzan a subrayar la importancia de dirigir la mirada hacia "hacia los variopintos y socialmente creativos arreglos que mujeres (y hombres) hacen para lograr que las personas de ellas/os dependientes sobrevivan y prosperen" (2008, p. 234). Estos arreglos incluyen, por una parte, todos aquellos hogares que rompen con el modelo convencional de familia nuclear, contra el que las feministas vienen luchando desde hace tanto tiempo y en el que cada vez vive una proporción menor de mujeres, pero por otra parte, también abarcan todo un conjunto de prácticas que tratan de situar la reproducción social y el cuidado en el marco de lo común (del Moral-Espín, 2012). Prácticas que, con frecuencia, se mueven en ese terreno resbaladizo que pone en relación las prácticas feministas con las que tienen que ver con los activismos pro-infancias (Rosen y Twamley 2018).

En concreto, las prácticas colectivas de crianza se configuran en este contexto de revitalización de lo comunitario como una esfera desde la que revalorizar la sostenibilidad de la vida y desde la que construir una alternativa a la configuración de la reproducción social. Entre ellas, han recibido mayor atención académica las centradas en la lactancia y grupos de madres (Faircloth, 2013; Massó, 2013; Villanueva, 2017) pero, en el contexto del estado español se observa también el desarrollo de iniciativas, orientadas a la primera infancia, en las que también participan varones (Araiza y González, 2016; Keller-Garganté, 2017; Elizalde y Díaz, 2021). En estas iniciativas tanto las tareas de cuidados como la gestión pedagógica son llevadas a cabo por grupos de madres y padres bajo esquemas participativos basados en la crianza con apego, solidaridad y la ayuda mutua, dinámica ajena a la empleada en las guarderías o en las opciones mercantiles. Ahora bien, cabría señalar que esta opción no surge (o al menos no únicamente) como una respuesta a la insuficiencia de la oferta pública o a las dificultades de accesibilidad, en términos monetarios, a las opciones del mercado. Aunque emergen a modo de crítica por como socialmente se organiza el cuidado, su gestación se asocia a una reacción hacia el modelo pedagógico que predomina en las tradicionales esferas de atención.

Tanto los estudios sobre las prácticas vinculadas al apoyo a la lactancia, como sobre los grupos de crianza enfatizan que la filosofía que sostiene estas experiencias está, en parte, vinculada a un concepto de maternidad denominado "maternidad intensiva" (Faircloth, 2013; Massó, 2013; Villanueva, 2017; Keller-Garganté, 2017; Elizalde y Díaz, 2021). Este término hace referencia a un modelo de ejercer la maternidad centrado en las niñas/ os que requiere dedicar grandes cantidades de tiempo, trabajo y dinero a la crianza con el fin, en la acepción original del concepto, de garantizar no solo el éxito de los niños/as en la vida sino también que destaquen por encima de sus compañeros/as de generación (Hays, 1996). El fenómeno ha ido ganando visibilidad e intensificándose, extendiéndose a la figura paterna (Ennis, 2014), de modo que con frecuencia se habla ya de "parentalidad intensiva" (Gómez-Espino, 2012) e incluso de "paternidad intensiva" (Shirani, Henwood y Coltart, 2012). El modelo de maternidad intensiva se asienta en una filosofía que pone en valor el apego y el vínculo materno-infantil y, en ocasiones, enlaza con un rebrote de discursos maternalistas esencialistas que reproducen una identidad tradicional de género legitimada a través de las necesidades de las criaturas. Por ello, es habitual que las mujeres que adoptan este modelo prolonguen su lugar en lo doméstico y abandonen el trabajo remunerado (en aquellos hogares que pueden permitírselo) para dedicarse plenamente al cuidado de hijas/os como fórmula de realización personal a través de un "trabajo de identidad" (Faircloth, 2013) que sigue entendiéndose como mandato "natural".

Para entender esta filosofía, es fundamental analizarla en relación con las transformaciones contemporáneas de los modelos reproductivos, de las estructuras 
familiares y del desarrollo del movimiento por los derechos de la niñez. Éste ha venido acompañado de una mayor consideración de las niñas/os como sujetos en sí mismos, un reforzamiento del valor dado a los lazos afectivos con los propios/as hijos/as y un debilitamiento de las nociones más tradicionales de autoridad. Sin embargo, por otro lado, también es esencial analizar la expansión de estos modelos de parentalidad en el marco neoliberal, marcado en la última década por el mandato austericida (Gálvez y Rodríguez, 2016), que ha acentuado la retirada del Estado de los ámbitos sociales y ha profundizado la precariedad en el empleo. En países como España, esta noción, puede tener un potencial analítico especialmente importante dado que a los valores familistas tradicionales se van sumando en las últimas décadas crecientes dosis de individualismo y competitividad. En este contexto, encuentra un perfecto asiento la focalización de la responsabilidad de los riesgos, la planificación y el bienestar social de niñas y niños sobre los hombros de familias e individuos (Vergara, Sepúlveda y Salvo, 2019). Estas filosofías sobre maternidades y parentalidad resultan de utilidad para abordar el estudio de los grupos de cuidados en la infancia que, como ya se ha señalado, al ser una práctica emergente, aún cuentan con un menor desarrollo teórico. Sin embargo, es fundamental distinguir la noción de "parentalidad" (parenting como sustantivo pero también como verbo) de la de crianza (Faircloth, 2013). Furendi (2002, citado en Lee, 2014) señala cómo la visión moderna de la actividad distintiva que hoy entendemos como "parentalidad" adquiere relevancia a partir del siglo XIX, cuando las niñas/os empiezan a considerarse una responsabilidad de su madre y padre en lugar de como una responsabilidad de una comunidad más amplia. De la misma manera, Hays (1996) sitúa la base de la cultura parental contemporánea en la separación entre "la familia" de las esferas más amplias de la economía y la sociedad que se produce a partir de la industrialización. Siguiendo esta línea podemos distinguir entre maternidad/parentalidad intensiva y "crianza con apego" que partiendo elementos similares (una crítica a los modelos de maternidad y crianza dominantes) apuesta por un carácter menos competitivo y más colectivo y politizado de la atención a niñas y niños.

Los trabajos de Araiza y González (2016) y de Keller-Garganté (2017), focalizados en el contexto de Barcelona, son posiblemente pioneros en la materia. En el último se definen los grupos de crianza compartida como "iniciativas comunitarias de resolución de cuidados en la primera infancia conformadas por familias que se autoorganizan para gestionar proyectos de crianza para sus hijas e hijos" (Keller-Garganté, 2017, p. 168). Si bien son iniciativas autónomas que presentan una gran heterogeneidad, la autora, resalta una serie de elementos compartidos en su muestra: la pequeña escala y propiedad colectiva, su carácter comunitario y autogestionado y los principios de cooperación, respeto, solidaridad, apoyo mutuo y horizontalidad que rigen su organización y funcionamiento. De ahí que su número haya aumentado desde el creciente interés por el paradigma de los comunes a partir de la última crisis económica mundial.

Las actividades parten de familias. Son éstas quienes toman las decisiones y las responsables de sostener el proyecto cotidianamente, aunque el nivel de implicación puede variar en función de si se cuenta o no, y en qué grado, con acompañantes remunerados. Su apuesta enlaza implícita o explícitamente con la reducción, redistribución y socialización de los cuidados que propone Federici (2013). Sin embargo, los análisis de la distribución efectiva de estas tareas entre madres y padres evidencia que ésta no es totalmente igualitaria y que, pese a los esfuerzos realizados, parece responder, cuantitativa y cualitativamente, a pautas tradicionales (Araiza y González 2016; Keller-Garganté, 2017). Gran parte del trabajo esencial para el mantenimiento de los grupos es realizado por mujeres bajo condiciones de gratuidad e incluso de precariedad. Este aspecto, nos recuerda que la externalización de los cuidados del espacio comunitario si bien visibiliza y revaloriza los cuidados no implica necesariamente una ruptura con la división sexual del trabajo (Vega, Martínez-Buján y Paredes, 2018). Para estos grupos sociales los cuidados se sitúan en el centro y, por ello, el 
trabajo reproductivo y de "cuidados de la naturaleza y de las personas siempre será más importante que el trabajo productivo-remunerado" (Araiza y González, 2016). En todo caso, este tipo de colectivos se deben entender como espacios afines a la economía feminista y a la propuesta de la sostenibilidad de la vida pues aúnan la filosofía de los "comunes" con la reproducción social contribuyendo a conceptualizar los cuidados como un bien común (Ezquerra, 2013). Veamos en los siguientes apartados cuáles son las características que presentan en el contexto de A Coruña.

\section{DISEÑO DE LA INVESTIGACIÓN Y METODOLOGÍA}

Los resultados de este artículo forman parte de la investigación "Cuidados en el ámbito comunitario. Experiencias, prácticas y vínculos para el sostenimiento de la vida en España y América Latina" (CSO2016-77960-R), financiada por el Ministerio de Ciencia, Innovación y Universidades durante el período 2017-2020. Dicho proyecto ha planteado como objetivo primordial analizar fórmulas de cuidados que se organizan y actúan en el polo comunitario $y$, entre ellas, las prácticas colectivas de atención durante la infancia constituían una de las acciones de interés por el aumento de su presencia en los últimos años. El diseño metodológico ha seguido una fundamentación cualitativa con la aplicación de técnicas de observación, entrevistas en profundidad semi-estructuradas y talleres de discusión entre las investigadoras y las participantes. Es decir, se ha adoptado un enfoque participativo.

Este artículo presenta una caracterización etnográfica de la ciudad de A Coruña y el trabajo de campo se ha realizado en la etapa enero 2018 - diciembre 2019. Para ello se procedió a analizar los espacios virtuales de comunicación y de interacción en los cuales estaban implicadas estas iniciativas. Este proceso supuso la clasificación de las experiencias según las siguientes categorías: actores involucrados, mediaciones (instituciones, mercado, familia), tiempo y duración, ámbito y contexto (local, barrio...), valores (misión), grado de institucionalización o informalidad y las actividades llevadas a cabo. A partir de esa información, se seleccionaron las iniciativas en las que se iba a realizar el trabajo de campo. Los criterios establecidos fueron dos: por una parte, que se tratasen de prácticas con un grado de estructura y de funcionamiento estable en el tiempo y, por otra, que programasen actividades continuadas con respecto a los cuidados. Es así como se eligieron los grupos "Canguras" y "Grupo de xogos" (Grupo de Juegos en castellano). Si bien se ha averiguado que cada uno de estos grupos no presenta un fuerte grado de organización interna y ésta se diluye entre sus integrantes, sí han podido identificarse las actrices claves de cada proyecto. A partir de ahí se contactó con las personas participantes de estos colectivos para la realización de las entrevistas en profundidad.

En total se han realizado 15 entrevistas tanto a personas que coordinaban las iniciativas de cuidados como a participantes en las mismas. El principal punto de referencia ha sido la voz, los discursos e imaginarios de personas protagonistas en las experiencias colectivas de cuidados, pero dichas narraciones han sido analizadas a partir de la involucración de las investigadoras en las comunidades objeto de estudio, de manera que, la técnica de la observación también ha guiado el trabajo de campo (Valles, 1997). Se ha concebido un diseño de muestreo flexible (Marshall y Rossman, 1989), lo cual significa que, si bien se ha partido de unas decisiones preestablecidas en los criterios de selección de las entrevistadas, también ha tenido cabida una visión circular de la investigación que ha dado lugar a reformulaciones a partir de los resultados obtenidos a lo largo del trabajo de campo (Valles, 1997). Ha sido la evolución del mismo el que ha indicado el número necesario de entrevistas a realizar en función del principio de saturación teórica (Glaser y Strauss, 1967). De la muestra seleccionada, 14 personas eran mujeres por lo que solamente se ha recogido 
el testimonio de 1 hombre. Este desequilibrio de género está motivado porque eran las propias mujeres las que estaban involucradas en mayor medida en las iniciativas escogidas $y$, por tanto, las conocedoras de primera mano del funcionamiento de los programas. Todas ellas tenían estudios universitarios y sus edades estaban comprendidas entre los 38 y los 47 años. En el análisis todas las entrevistas están anonimizadas y los nombres que se utilizan en el análisis no son reales.

Las entrevistas se realizaron personalmente por las autoras del artículo con una duración de entre 60-90 minutos y todas han sido grabadas y transcritas. Se utilizó un guión que permitió mantener una conversación estructurada sobre los temas de interés focalizados en las fórmulas de organización del grupo, la participación de los integrantes, las actividades que realizan, los debates internos, las iniciativas de colaboración con otros agentes del entorno y las relaciones entre los miembros. Sin embargo, la conversación siguió un enfoque participativo en el que las entrevistadas podían incluir aquellas cuestiones que estimasen de relevancia.

Finalmente, se estableció un nivel de participación más amplio con las informantes a través de la realización de dos talleres cuya finalidad consistía en analizar en común los materiales recabados durante el trabajo de campo y recabar un discurso colectivo que complementase a las experiencias personales. Estos talleres estuvieron coordinados por las autoras del artículo, y a todas las entrevistadas se les ofreció la posibilidad de participar, aceptando 14 de ellas. De esta manera, estos talleres se convirtieron en un espacio de comunicación con el equipo de investigación y de intercambio de ideas y prácticas.

\section{GÉNESIS, FUNCIONAMIENTO Y RELACIONES EN LAS EXPERIENCIAS COLECTIVAS DE CRIANZA}

Las prácticas colectivas de cuidados, como ya hemos mencionado, son experiencias cooperativas de crianza entre grupos de madres y padres que, mediante relaciones de reciprocidad y ayuda mutua, comparten las tareas de atención a los menores durante la primera infancia (de 0 a 3 años). Para ello, es indispensable habilitar un espacio de confluencia y poseer una cierta infraestructura organizativa que permita una adecuada información sobre los encuentros y las actividades a realizar en los mismos.

El trabajo de campo realizado en A Coruña muestra una elevada heterogeneidad entre estas iniciativas según su grado de institucionalización, modos de colaboración y funcionamiento. En términos generales, destaca que ninguna de las experiencias identificadas mantiene elevados niveles de formalización y no se acercan al nivel institucional encontrado en otras ciudades. Por ejemplo, en el análisis de Keller-Garganté (2017), se identifican para el caso de Barcelona grupos constituidos de crianza compartida que suponen verdaderas alternativas de cuidados con respecto a las guarderías o a la contratación privada de cuidadoras en domicilios. La diversidad de A Coruña puede estar vinculada al carácter reciente tanto de la expansión de los grupos feministas y de los movimientos más críticos en el ámbito de los cuidados, como de las experiencias de autogestión. Ambos procesos se han consolidado en la última década por lo que las prácticas colaborativas de crianza representan una revitalización de valores y simbologías que no estaban históricamente ancladas en este territorio. En otros espacios, como Barcelona, las iniciativas de apoyo mutuo están fuertemente enraizadas en el asociacionismo local, fenómeno ampliamente documentado por la literatura académica (Carbonell, Gálvez y Rodríguez-Modroño, 2014). En el caso de A Coruña, las evidencias del campo señalan que si bien se trata de prácticas solidarias que aportan una identidad colectiva a sus integrantes con elementos comunes de afinidad que permiten dibujar un modelo, la colectividad 
únicamente funciona en momentos muy concretos que suelen estar asociados al ocio y al tiempo libre. Es decir, carecen de una estructura organizativa de cuidados cotidianos que las dote de potencial para ser una elección independiente frente a las otras esferas públicas y privadas bajo las que se organiza socialmente la crianza.

Los discursos de sus integrantes señalan que estas experiencias aparecen a partir de su involucración en grupos feministas con una visión crítica hacia valores hegemónicos sobre la maternidad. Éstos incluyen tanto las prácticas médicas hacia el parto como las creencias y valores sobre las que se ha configurado la crianza y la educación de los menores. De esta manera, todas las personas entrevistadas habían formado parte de la asociación "O parto é noso" (El parto es nuestro, en castellano), una agrupación que ofrece información y asesoramiento a mujeres embarazadas en cuestiones del parto y el puerperio. Todas coinciden en confirmar que esta asociación las dotó de un sentimiento de pertenencia y de refugio en donde poder debatir valores propios sobre la maternidad ajenos a los que les ofrecian otras mujeres de su entorno. Una vez que nace el bebé también se cuestionan aspectos básicos de la crianza hegemónica como la utilización de guarderías desde el cuarto mes de vida, los tiempos de duración del trabajo remunerado, el alejamiento de la madre y el bebé a partir de las 16 semanas que dura la baja de maternidad, la centralidad de la posición del adulto con respecto a las necesidades y requerimientos de las criaturas en los modelos institucionales...Y es así como se visualiza la existencia de otro nexo de unión entre las componentes de las experiencias colectivas de cuidados: su participación activa en foros virtuales sobre crianza natural, adhesión que surge vinculada a la necesidad de compartir con iguales sus experiencias sobre la crianza alejadas de la cultura convencional.

En este sentido, el discurso más aclamado por todas las mujeres entrevistadas es que la puesta en marcha de prácticas de cuidados colaborativas estuvo influida especialmente por el sentimiento de soledad que se produce durante los primeros años de vida de los pequeños/as y por la falta de espacios públicos y políticos en los que compartir las inquietudes que surgen durante maternidad y la crianza, sobre todo, cuando no se siguen las pautas mayoritarias. En términos generales, los supuestos básicos de crianza que se defienden están basados en el vínculo materno-filial, la lactancia prolongada y extensiva, el colecho y el porteo (Villanueva, 2017).

Una crianza donde se respete más al niño, su ritmo, sus necesidades y ahí es donde estás todavía más sola. Porque claro, entonces, tus amigas te empiezan a decir que estás en una jaula, que qué es eso de que mande el niño, que tal. Por ejemplo, es dar teta hasta que el bebé se destete, o no ir de cumpleaños porque tienes un bebé de un año que tome teta. Amigas que de repente dicen: "Pero bueno, ¿cuándo vas a salir, cuando vas a hacer tu vida?", "Pues no lo sé. ¡Dentro de un año o 22! (Nieves, 44 años, 2 hijos).

Ello conlleva a centrar la crianza en las necesidades de los niños/as, considerar que el "buen cuidado" es aquel que procura que éstos estén la mayor parte del tiempo con las familias evitando horarios extensivos en guarderías o con personas ajenas y en donde la educación es guiada por los propios pequeños/as. Es decir, elementos del modelo descrito anteriormente como "maternidad intensiva" pero inculcándole un vínculo comunitario. En ese proceso de demanda de un nuevo tipo de crianza nuestras informantes evocan un regreso al modelo anterior al capitalismo industrial, con una mayor responsabilidad de la comunidad en el cuidado de las criaturas. Por ello reaccionan ante la generación del biberón, la familia nuclear, las malas prácticas de conciliación, el trabajo productivo exigente con horarios tasados, la escolarización temprana etc. No es casual que estas mujeres encuentren en no pocas ocasiones dificultades para ser comprendidas por sus madres, y en cambio hagan frecuentes referencias a las abuelas como mejores prescriptoras, y más cercanas en sus métodos de crianza. 
Las fórmulas institucionalizadas de cuidados y educación tampoco cumplen con las características demandadas así como tampoco lo hace el mercado privado orientado en España hacia la contratación de empleadas de hogar. Es más, los grupos compartidos de crianza se fundamentan en una crítica a este modelo de atención: "No teníamos intención de meterlos en guarderías y seguir con nuestra vida. Que es lo que te dicen que hay que hacer. Seguir con tu vida normal, ir al gimnasio... como si hubieras tenido en vez de un niño un perro o un peluche" (Sandra, 45 años, 2 hijos). Paradójicamente en esa renuncia del modelo convencional de cuidados es cuando aparecen tensiones con las convicciones feministas de las participantes. La maternidad intensiva las coloca en una situación de vuelta al hogar, de aislamiento y de cierta subordinación económica respecto del varón. A esto hay que sumarle una sobrepresencia de las madres en el proceso de crianza y un cierto repliegue de los varones al menos durante los primeros años de cuidados. Nuestras informantes viven el proceso con un protagonismo indiscutible de ellas mismas, así que podríamos decir que son maternidades que en todo el proceso de embarazo-parto-crianza natural la figura del padre-varón aparece diluida. Los varones no son introducidos en el debate por las mujeres entrevistadas, no aparecen como críticos del modelo ni tampoco como defensores del mismo. Las tensiones que se generan sobre las formas de criar son vividas únicamente entre mujeres pro y contrarias a esta filosofía.

\begin{abstract}
Casi todas las mujeres, muchas mujeres de El Parto es Nuestro, muchas mujeres de Canguras, muchas doulas, dejaron sus trabajos previos por maternidad. Los padres iban a trabajar, tienen su espacio, su aire, su no sé qué. Porque un primer vínculo es con la madre, entonces creo que tenemos que reivindicar desde nuestro feminismo esa figura. Hay un lazo muy poderoso que une a los niños, niñas y a sus padres, y sobre todo a sus madres. Un espacio que, aparte, nadie más que yo puede hacer. Entonces, creo que tenemos que reivindicar desde el feminismo esa figura (Nieves, 44 años, 2 hijos).
\end{abstract}

Aparentemente, los padres varones se muestran solícitos y dispuestos a cumplir con todas las prerrogativas que requieren las criaturas y sus madres. Muchos se convierten en acompañantes comprometidos con este modelo, estando presentes en un segundo plano en un principio, y adquiriendo protagonismo a medida que los pequeños/as iban creciendo y descendía la dependencia materna. Podemos considerar que en estos casos se cumplen con los criterios de una nueva paternidad involucrada afectivamente y que además demanda la presencia de lugares colectivos desde los que reclamar su figura. Pero también es cierto que se detalla la vivencia de muchas tensiones en las parejas y que algunos varones se sienten incomprendidos en esta etapa de paternidad e incluso perdidos, pues o bien no saben cómo ejercer este rol, o bien no comprenden la filosofía con la que sus parejas femeninas están ejerciendo la crianza.

Para que te hagas una idea, la abuela habrá quedado por necesidad con ellos unas 3 veces o 4 desde que han nacido, desde hace ocho años. Y eso en los últimos 2 años. Y claro, eso te carga. Es mucho, es muchísimo, y es a costa de nuestra salud, de nuestra relación que ha estado en la cuerda floja millones de veces, por desgracia, porque no podemos aguantar esa presión, es durísimo, pero por otro lado yo soy incapaz de ir en contra de todo esto que he descubierto, son...principios (Sandra, 45 años, 2 hijos).

Aunque se detectan estas pautas esencialistas sobre el papel de las mujeres-madres estas experiencias se gestan desde reflexiones críticas con la organización social del cuidado imperante: "Yo lo que reclamo es tener la centralidad cuidando. Porque a nosotras sólo se nos permite tener la centralidad en la medida en que nos pleguemos a los patrones masculinos tradicionales" (Maite, 37 años, 2 hijos). Y es ahi donde se evoca la necesidad de afianzar la comunidad, los grupos de pares y el entorno. Es en ese momento cuando se posicionan en contra del individualismo y de la competitividad del sistema educativo 
que no fomenta valores de solidaridad y cuando se recurre a la idea de generar nuevas fórmulas de ayuda mutua como vía alternativa desde las que tejer la existencia. Porque dichas prácticas están comprometidas activamente con situar a los cuidados en el centro del sistema y según esta filosofía esto requiere distanciarse de la lógica productivista que pone los beneficios por encima de las personas.

Precisamente, uno de los grupos analizados, el denominado "Grupo de Xogos" surge a partir de las cavilaciones de varias de sus componentes sobre estas ideas. La principal dificultad que encontraron fue la de buscar un espacio que permitiese tramar estas redes en común entre las familias y es así como se accede a un espacio ocupado en la ciudad de A Coruña, el cual ya estaba integrado en uno de sus barrios. La ilusión de comenzar un nuevo proyecto en común unió fuerzas entre los progenitores que adaptaron el espacio para convertirlo en un lugar de encuentro, un lugar educativo y de ocio en el que poder programar actividades con los menores y donde los progenitores y especialmente las madres encontraban un espacio de apoyo.

\begin{abstract}
Entonces con una amiga hablamos de la idea de montar algo más formal. Y empezamos a darle forma a esa idea de montar un grupo de juegos que después se llamó Grupo de Xogos. Y coincidió además que estaba recién ocupado el Centro de Palavea y allí nos ofrecieron un espacio a partir de un chico que conocíamos. [...] El espacio estaba guay así que fuimos un día con una radial e hicimos unas puertas redondas, un sitio para gateo, había una madre que tenía unas alfombras gigantes que también nos las dejó... Empezamos a llevar cosas e hicimos todo en común (Lidia, 38 años, 1 hija).
\end{abstract}

Puede comprobarse, por tanto, que en el comienzo de esta experiencia se encuentra una coincidencia generacional y vital de mujeres feministas militantes, las cuales están asociadas también a otras redes de activismo como puede ser la defensa de la naturaleza y el reclamo de un cambio en la organización del sistema económico. El éxito de la iniciativa animó la participación de alrededor de 40 familias y el modo de funcionamiento en sus comienzos fue el de programar encuentros dos días a la semana que se ampliaron con el tiempo y de comunicarse a través de plataformas digitales. Se creó un blog y una lista de correo electrónico que llegó a aglutinar a unas 150 familias. A partir de esos canales se proponían actividades a realizar con los pequeños/as y se recaudaban los materiales necesarios para estas tareas (instrumentos musicales, cuentos, material de reciclaje para manualidades...) así como se convertía también en una línea abierta de debate sobre modelos educativos, pediatras, películas, libros... Es decir, circulaban saberes y opiniones entre los miembros integrantes y siempre las mujeres fueron la parte más activa. Desde el entorno virtual se programaban los encuentros y las actividades y la intensidad en la participación partía de los deseos individuales de cada familia y de su grado de involucración.

La heterogeneidad del grupo era amplia en términos ideológicos: desde punkis y anarquistas hasta católicos. Las integrantes destacan como elemento positivo esta falta de uniformidad entre las familias y también la conexión que tenían con el barrio en el que se integraban. Pudieron crear más tareas colectivas que ampliaban el concepto de crianza hacia el de sostenibilidad de la vida como el mantenimiento de un huerto urbano con la ayuda de los vecinos/as del entorno. Éstos también acudían al centro ocupado a realizar diversos talleres y eso nutría la crianza con la vida que transcurría a su alrededor. La gestión se realizaba de manera participativa con reuniones y asambleas en donde se priorizaban los valores de solidaridad y respeto. De esta manera, las relaciones que se generaron a través de esta colaboración fueron muy intensas en términos afectivos y personales. En gran parte porque en la organización era esencial una elevada presencia de los/as integrantes y de su participación. Destaca el papel protagonista de las mujeres tanto en los debates como en la gestión. A pesar de este nivel de horizontalidad, cabe 
mencionar que el mayor peso de administración tanto de las aplicaciones informáticas como de los espacios y de las actividades partía fundamentalmente de seis familias. Ha sido una constante entre las entrevistadas destacar las dificultades de mantener una coordinación activa y, aunque existían elevados niveles de voluntariedad, la dinámica de participación finalmente solía recaer sobre las mismas personas, situación que provocó tensiones entre los/as integrantes.

El grupo se diluyó al cabo de tres años cuando se desocupó el espacio por parte de las autoridades legales. Intentaron buscar otras opciones pero no encontraron ningún lugar apropiado. Los centros cívicos no disponían de capacidad para albergarlos. A eso se suma el crecimiento de los pequeños/as y su escolarización en la mayor parte de los casos junto con el propio desgaste que supuso la implicación intensiva y participativa. Las narraciones coinciden en que éstos han sido también los motivos por lo que las otras experiencias han dejado de funcionar sin que existiese un relevo generacional en los proyectos. La coincidencia temporal de sus inicios en la etapa 2008-2010, en plena crisis económica y de (re)arme de los principios colectivizadores en España, podría indicar que el contexto político y económico también han sido fuente de inspiración al encontrar afinidades en los movimientos sociales de la ciudad y en los propios gobiernos municipales. Es el caso de Madrid, en donde, a partir del programa Mares promovido por la corporación municipales, se potenciaron formaciones y talleres dirigidos a la creación de grupos de crianza compartida (Elizalde y Díaz, 2021).

\section{DEBATES, TENSIONES Y RUPTURAS CON EL SISTEMA EDUCATIVO}

La homogeneidad de posiciones que caracterizó la etapa de crianza, con respecto a los valores que rodeaban la maternidad y la parentalidad, se convierten en opciones dispares y a menudo incompatibles entre sí cuando las familias se enfrentan a la decisión de escolarizar a sus hijos/as. La disyuntiva surge alrededor de los tres años de vida de los pequeños/as aunque en ocasiones se retrasa hasta la edad obligatoria de los seis años. Básicamente, el debate se centra en el modelo educativo por el cual optar. La mayoría de las familias integrantes se decantan por la educación oficial en centros públicos o privados, pero, una parte fuerte del grupo decide contrastar opciones con modelos pedagógicos libres que albergan tanto escuelas creadas por grupos de familias como las prácticas del homeschooling (educación en casa guiada por los progenitores) y el unschooling (sin una educación controlada por los progenitores siendo los pequeños/as los que deciden las cuestiones de estudio según sus intereses y capacidades). La intensidad del debate llega a convertirse en una fuente de tensión entre los/as participantes por lo que la elección por uno u otro modelo terminó por dispersar al colectivo e incluso redujo la intensidad de las relaciones entre ellos/as.

En términos generales, las familias que deciden optar por una educación alternativa coinciden con aquellas en las que la práctica de la maternidad intensiva fue más exaltada y esencialista. De esta manera, es el vínculo con la madre y los deseos de ésta de estar presente en la vida cotidiana del pequeño/a una de las poderosas razones por las que se esquiva la educación formal. Así lo comenta el único varón entrevistado, cuyo discurso refleja esta visión maternalista que delega a las madres la responsabilidad de este proceso:

Hay un lazo muy poderoso que une a los niños, niñas y a sus padres, y sobre todo a sus madres. Entonces suele ser la mujer la que dice: "No, no, no, al colegio no va". Porque suele ser la mujer la que con el parto en casa, con los grupos de lactancia, establece un vínculo poderosísimo con la criatura, y llegan los 3 años, que es la edad típica en la que la gente se plantea escolarizar y en ese momento con ese vínculo dice: 'No' (Juan, 41 años, 1 hija). 
Además de este componente emocional, los motivos para no escolarizar de manera formal también se nutren de componentes ideológicos; las actitudes críticas hacia la pedagogía de los colegios convencionales suele ser otro de los elementos primordiales. En este caso, el nivel de descontento varía en cada familia aunque prácticamente está relacionado con la asimilación de la escuela a un modelo de enseñanza diseñado en la etapa industrial anquilosado en la disciplina y el control. El nivel de intervención educativa se considera perniciosa y poco respetuosa con las capacidades, deseos y circunstancias individuales de los niños/as.

Además, resaltan que quieren educar a sus hijos/as en valores en los que se potencie la reflexión crítica, la cultura democrática y asamblearia, y en donde la naturaleza se integre como una dimensión básica para la sostenibilidad humana y ello lo ven incompatible con la escuela formal. Tal y como hicieron con su involucración en el grupo de crianza buscan otros lugares de aprendizaje desde los que expresar una acción que no esté dominada por las lógicas que marca la administración pública o el mercado privado. Sus discursos narran disconformidades con aquellos modelos educativos que únicamente muestran opciones políticas predeterminadas y desde los cuales se intenta que los pequeños/as se conviertan en seres socialmente controlables con roles políticos y económicos esperados. Es así como estas opciones desafían el paternalismo tradicional de la educación que se fundamenta en el no reconocimiento de los derechos y autonomía de los niños/as (Pechtelidis, 2018).

\begin{abstract}
Por qué me tienen que organizar a mi la institución educativa, me tienen que organizar la vida ¿no? El vecinito de al lado tiene siete años y ya tiene deberes. Los fines de semana muchas veces no podemos quedar porque tiene que hacer deberes con siete años ¿eh? O sea, eso es esclavitud, que un niño de siete años... Y vemos los resultados, vemos a un montón de niños medicados por TDH, porque no les dejan ser niños (Susana, 45 años, 2 hijos).
\end{abstract}

Por ello, algunas de las familias entrevistadas incluso llegaron a fundar escuelas alternativas basadas en los principios de educación libre (emancipación de los niños/ as con respecto de los adultos educadores). El hecho de que finalmente esta opción sea privada y requiera de una inversión económica por parte de los/las integrantes provoca que se convierta en una estrategia de clase social media o alta con ingresos elevados y/o con empleos flexibles en horarios. Homeschooling o unschooling aunque son prácticas más económicas en la realidad también se configuran como vías limitadas si se tiene en cuenta la precariedad e intensidad laboral que exige el mercado laboral actual es complicado que se den las circunstancias para la escolarización en el hogar. A nuestro entender, estas experiencias son realmente desafiantes en términos políticos pero, sin embargo, también promocionan una comunidad en cierta medida privilegiada que no revierte ni las desigualdades de clase ni de género. Las escuelas libres creadas están apartadas de la ciudad en la que habitan las familias porque sus proyectos chocan con la legalidad establecida y esta misma situación sucede también con las prácticas de no escolarización. Que sean consideradas ilegales, las aparta del territorio en el que los pequeños/as residen $y$, por tanto, parecen contribuir a una educación exclusiva de algunos grupos sociales.

La ruptura con el modelo pedagógico de las escuelas convencionales atraviesa también los discursos de las informantes que finalmente han escolarizado a sus hijos/as en los colegios públicos. Las limitaciones que encuentran coinciden con las ya explicadas: la uniformización, la escasa atención a la diversidad, la ausencia de participación activa de los niños/as... Sin embargo, desde esta posición crítica también parecen surgir fórmulas de resistencia. La más relevante en términos de capacidad de transformación social ha sido el desarrollo de una estrategia de "entrismo". Es decir, ocupar los espacios convencionales educativos para cambiarlos desde dentro. Presionar para que la institución educativa vaya asumiendo preceptos y prácticas de crianza más respetuosa. 
Lo que tenemos es lo público, ¿sabes? Es lo que hay. Entonces hay que cambiarlo desde dentro (Mabel, 37 años, 2 hijos).

La escuela también la construimos entre todas. Si estamos, también tenemos la responsabilidad (Juana, 37 años, 2 hijos).

Es así, como otro grupo de familias entrevistadas decidió desplazar su activismo hacia el barrio en el que viven asumiendo la dirección del AMPA de un colegio público en el que han escolarizado a sus hijos, gestionando también la Asociación de Vecinos de esa zona y activando redes entre diversos agentes como comerciantes, familias, seguridad local... para que pueda cohesionarse una comunidad que, en gran parte, se ha perdido en las últimas décadas. De esta manera, ya han recuperado tradiciones festivas típicas del barrio (romerías), han realizado un documental histórico de su evolución, han modificado las normas de juegos en el patio del colegio para que sean inclusivas en términos de género, han creado una ruta segura que los niños/as puedan ir solos/as caminando al colegio y organizan actividades intelectuales y lúdicas con otras escuelas y bibliotecas municipales. En este caso, la idea es "transformar el barrio" partiendo de un espacio físico común reconocible para la relación de pares, aspecto que, según sus integrantes, fue una de las razones esenciales por las que no llegaron a sobrevivir los grupos de crianza analizados. El barrio aparece como una nueva forma de lo común desde la cual enfocar una ciudadanía política preservando sus instituciones como las escuelas. En este sentido, lo público se convierte en una dimensión desde la cual se puede construir comunidad.

Porque todos esos vínculos sociales, las relaciones afectivas es una riqueza. Un proyecto local de este tipo tiene mucha más diversidad que las prácticas privadas de no escolarizar que no dejan de ser opciones de privilegio (Mabel, 37 años, 2 hijos).

\section{CONCLUSIONES}

Este artículo parte de las reflexiones en torno a la organización social del cuidado generadas durante la última década a partir de las conexiones entre activismo y academia feminista. Para ello, se retoma una noción de comunidad en el sentido que había sido otorgado por las corrientes feministas-marxistas de los años 70 y se pone en diálogo con los planteamientos derivados de nuevas filosofías de crianza y maternidad-parentalidad y su relación con la transformación de los modelos reproductivos, de las estructuras familiares y del desarrollo del movimiento por los derechos de la niñez. En este sentido, el análisis demuestra que el cuidado como una actividad colaborativa se está configurando como un ámbito específico, pero no aislado de las otras esferas de provisión (a saber, Estado, mercado y familia), y que éste mantiene una elevada heterogeneidad en cuyo desarrollo influyen las propias dinámicas territoriales cambiantes. En este marco, el análisis de prácticas situadas desde las que se traman cuidados en común son esenciales para comprender los aportes del ámbito comunitario a su organización social.

En concreto, el documento ha caracterizado la composición de los grupos de crianza compartida, las condiciones sociales y políticas de su emergencia, así como las rupturas que simbolizan con respecto a la tradicional organización social del cuidado. Estas experiencias se han analizado a partir de un trabajo de campo cualitativo. El análisis de los discursos y narraciones sugieren que estas iniciativas enfatizan el actuar en conjunto como elemento principal para su devenir, siendo esta colectividad la que genera sentido, valores y afectos que, en definitiva, convierten a esas prácticas en una comunidad resguardada del lucro individual y situada más allá del mercado. De esta manera, consiguen desplazar la organización social del cuidado hacia la esfera de lo "común". 
Los resultados confirman que estos grupos de crianza suponen un cambio generacional relevante en los ámbitos de la maternidad y de la infancia en cuanto desafían las posiciones que las personas adultas y los niños/as ocupan tanto en el orden social como económico. Su filosofía de actuación se basa en la centralidad de los cuidados y su primacía frente a las relaciones productivas. Pero esta postura genera fuertes tensiones en dos sentidos.

Por una parte, con el activismo feminista de sus integrantes que han desplazado los intereses de reivindicación desde la igualdad en el plano laboral hacia el desarrollo con plenitud de sus facetas como madres, considerando esta tarea indispensable para el sostenimiento de la vida. Estos resultados coinciden con los identificados en la literatura analizada en el marco teórico que también subraya como ello conlleva contradicciones relacionadas con la filosofía de la maternidad intensiva por el contenido esencialista de estos preceptos tanto en términos de género, porque se afianza la propia división sexual del trabajo, como de clase social, al sentir que sólo personas con elevada flexibilidad laboral pueden romper con los tiempos estipulados de cuidados y trabajo. En todo caso, sus integrantes parecen encontrar nuevas oportunidades de relaciones sociales no capitalistas que inundan también a sus compañeros varones, lo cuales, comienzan a despegar valores de una paternidad afectiva que choca con el planteamiento de generaciones anteriores. Eso sí, este proceso no está ausente de tiranteces e incluso de rupturas entre las parejas.

Por otra parte, las tensiones también se encuentran en relación con el modelo educativo convencional. En cuanto los niños/as alcanzan la edad obligatoria de escolarización estas familias demandan alternativas de enseñanza que supongan un ruptura con la disciplina y autoridad de la oferta convencional. De ahí su compromiso en la creación de escuelas libres y en la práctica de homeschooling o unschooling. El alejamiento de estos progenitores del sistema escolar formal puede considerarse como un nuevo modelo de gobernanza de la niñez que pone el acento en el derecho de las criaturas a tomar decisiones y participar activamente en el proceso de aprendizaje, en línea con los movimientos pro derechos y autonomía de la infancia. Sin embargo, en estas opciones se advierten escasas posibilidades de cambio en la vida pública y colectiva ya que se convierten en espacios privilegiados para ciertos grupos sociales con unas condiciones laborales flexibles y/o de altos ingresos.

De momento, en lo que atañe a nuestro trabajo de campo, la experiencia más transformadora en términos de apertura, justicia social y bienestar de la comunidad está relacionada con una estrategia desplegada desde dentro del sistema público de enseñanza. Se trata de ocupar los espacios de dirección de los colegios públicos a través del AMPA y del barrio, sobre todo, a partir de la activación de las asociaciones vecinales. Mediante estas instituciones el objetivo es recuperar la cohesión comunitaria y modificar las pautas tradicionales de enseñanza.

En términos generales, las mujeres han experimentado a través de los grupos de crianza nuevas maneras de pensar y actuar que las ha comprometido en relaciones sociales alternativas a las que ofrece el sistema formal. De esta manera, estas comunidades pedagógicas ofrecen la posibilidad de fomentar una actividad política que se engendra desde las propias tareas educativas y de crianza. 


\section{REFERENCIAS}

Araiza, A. y González, R. (2016). Hacia la colectivización del cuidado. La Mainada, una experiencia de crianza compartida. Otra Economía, 10(19), 176-184.

Barbeta-Viñas, M. y Cano, T. (2017). ¿Hacia un nuevo modelo de paternidad? Discursos sobre el proceso de implicación paterna en la España urbana. Revista Española de Investigaciones Sociológicas, 159, 13-30.

Barbeta-Viñas, M. (2019). ¿Una paternidad no tan nueva? Los discursos de jóvenes y padres sobre la implicación paterna: una comparación. Revista Española de Sociología, 28(1), 9-26.

Carbonell, M., Gálvez, L. y Rodríguez-Modroño, P. (2014). Género y cuidados: respuestas sociales e institucionales al surgimiento de la sociedad de mercado en el contexto europeo. Áreas, 33, 17-32.

Carrasco, C. (2016). Sostenibilidad de la vida y ceguera patriarcal: una reflexión necesaria. Atlánticas: revista internacional de estudios feministas, 1(1), 34-57.

Dalla Costa, M.R. y James, S. (1972). El poder de la mujer y la subversión de la comunidad. México D.F.: Siglo XXI.

Daly, M. (2020). Children and their Rights and Entitlements in EU Welfare States. Journal of Social Policy, 49(2), 343-360.

Del Moral-Espín, L. (2012). En transición. La epistemología y filosofía feminista de la ciencia ante los retos de un contexto de crisis multidimensional. E-cadernos ces, 18, 51-80. https://journals.openedition.org/eces/1521

Elizalde-San Miguel, B. y Díaz, M. (2021). Grupos de crianza comunitaria: ¿iniciativas comunitarias o grupos identitarios? Revista Española de Sociología, 30(2), a30. https://doi.org/10.22325/fes/res.2021.30

Ennis, L.R. (ed) (2014). Intensive Mothering: The Cultural Contradictions of Modern Motherhood. Toronto: Demeter Press.

Ezquerra, S. (2013). Hacia una reorganización de los cuidados: ¿entre lo público y lo común? Viento Sur, 130, 78-88.

Faircloth, C. (2013). Militant Lactivism? Attachment Parenting and Intensive Motherhood in the UK and France. Nueva York: Berghan Books.

Federici, S. (2013). Revolución en punto cero. Trabajo doméstico, reproducción y luchas feministas. Madrid: Traficantes de Sueños.

Flaquer, Ll., Navarro-Varas, L., Antón-Alonso, F., Ruiz-Forès, N. y Cónsola, A. (2018). La implicación paterna en el cuidado de los hijos en España antes y durante la recesión económica. Revista Española de Sociología, 28(2), 249-268.

Gálvez, L. y Rodríguez-Modroño, P. (2016). Una crítica desde la economía feminista a la salida austericida de la crisis. Atlánticas. Revista Internacional de Estudios Feministas, 1(1), 8.

Gelb, A. (1988). Oil windfalls - Blessing or curse? Washington, D.C.: World Bank Research.

Glaser, B. y Strauss, A. (1967). The discovery of grounded theory. Chicago: Aldine.

Gómez-Espino, J.M. (2012). Two sides of intensive parenting: Present and future dimensions in contemporary relations between parents and children in Spain. Childhood, 20(1), 22-36. 
Graham, H. (1997). Feminist perspectives on caring. En J. Bornat, J. Johnson, C. Pereira, D. Pilgrim y F. Williams (eds.), Community Care a Reader (pp. 124-133). London: MacMillan Press.

Hardin, G. (1968). The tragedy of the commons. Science, 162, 1243-1248.

Harding, S. (2008). Sciences from Below: Feminisms, Postcolonialities, and Modernities. Durham: Duke University Press.

Hays, S. (1996). The cultural contradictions of motherhood. New Haven: Yale University Press.

Keller-Garganté, C. (2017). Grupos de Crianza Compartida: Una alternativa comunitaria en la organización del cuidado en la primera infancia. Quaderns de l'Institut Catala d'Antropologia, 22(2), 167-82.

Kioupkiolis, A. y Pechtelidis, Y. (2018). Youth heteropolitics in crisis-ridden Greece. En S. Pickard y J. Bessant (eds.), Young People Re-generating Politics in Times of Crises (pp. 273-293). Cham: Palgrave Macmillan.

Laval, C. y Dardot, P. (2015). Común. Ensayo sobre la revolución en el siglo XXI. Barcelona: Gedisa.

Lee, E.J. (2014). Introduction. En E. Lee, J. Bristow, C. Faircloth y J. Macvarish, Parenting Culture Studies (pp. 1-24). London: Pallgrave Macmillan.

Marshall, C. y Rossman, G. (1989). Designing Qualitative Research. Newbury Park, California: Sage.

Massó, E. (2013). Lactancia materna y revolución, o la teta como insumisión biocultural: calostro, cuerpo y cuidado. Dilemata, 5(11), 169-206.

Moreno Mínguez, A., Ortega Gaspar, M. y Gamero, C. (2017). Los modelos familiares en España: reflexionando sobre la ambivalencia familiar desde una aproximación teórica. Revista Española de Sociología, 26(2), 149-167.

Ostrom, E. (1991). Governing the Commons: The Evolution of Institutions for Collective Action. New York: Cambridge University Press.

Pechtelidis, Y. (2018). Heteropolitical Pedagogies: Chitizenship and Childhood. Commoning Education in Contemporary Greece. En C. Baraldi y T. Cockburn (eds.), Theorising Childhood. Citizenship, Rights and Participation (pp. 215-237). Cham: Palgrave Macmillan.

Pérez-Orozco, A. (2014). Subversión feminista de la economía: aportes para un debate sobre el conflicto capital-vida. Madrid: Traficantes de Sueños.

Rendueles, C. y Subirats, J. (2016). Los (bienes) comunes. ¿Oportunidad o espejismo? Barcelona: Icaria.

Rosen, R. y Twamley, K. (2018). Feminism and the Politics of Childhood-Friends or Foes? Londres: UCL Press.

Shirani, F., Henwood, K. y Coltart, C. (2012). Meeting the Challenges of Intensive Parenting Culture: Gender, Risk Management and the Moral Parent. Sociology, 46(1), 25-40.

Valles, M. (1997). Técnicas cualitativas de investigación social: reflexión metodológica y práctica profesional. Madrid: Síntesis.

Vega, C. y Martínez-Buján, R. (2017). Explorando el lugar de lo comunitario en los estudios de género sobre sostenibilidad, reproducción y cuidados. Quaderns, 22(2), 65-81. 
Vega, C., Martínez-Buján y R, Paredes, M. (2018). Introducción. Experiencias, ámbitos y vínculos cooperativos para el sostenimiento de la vida. En C. Vega, R. Martínez-Bujan y M. Paredes (eds.), Cuidado, Comunidad y Común (pp. 15-50). Madrid. Traficantes de Sueños.

Vergara, A., Sepúlveda, M. y Salvo, I. (2019). Being a parent and being a child in Chile today: the relational construction of subject positions in a neoliberal context. Subjectivity, 12(4), 371-88.

Villanueva, D. (2017). La maternidad glorificada: análisis de los discursos sobre maternidad de mujeres que participan en grupos de crianza respetuosa de la región de Valparaíso, Chile. Revista Punto Género, 7, 138-155. 